\title{
Obtención industrial de materiales cerámicos a partir de lodos rojos del proceso Bayer
}

\author{
G. A. PÉREZ ROdRíGueZ*, F. GUITIÁN RIVERA*, S. DE AZA PENDÁS** \\ *Instituto de Cerámica de Galicia (ICG). Edificio Monte da Condesa, Campus universitario Sur. 15706 Santiago de Compostela. A Coruña \\ **Instituto de Cerámica y Vidrio (ICV).Km 24,3 Carretera Antigua Madrid-Valencia, 28500 Arganda del Rey (MADRID)
}

\begin{abstract}
Los Lodos Rojos son el residuo insoluble de la extracción de alúmina a partir de la bauxita por el proceso Bayer. En Galicia se producen más de 400.000 toneladas anuales de este residuo industrial, constituido fundamentalmente por una mezcla de óxidos e hidróxidos de hierro, óxidos de titanio, cuarzo, con cantidades menores de, arcillas, y otros minerales.

El presente trabajo describe el proceso de aprovechamiento industrial de los Lodos Rojos, para obtener ladrillos densos para acumuladores de calor, áridos para carreteras y otros productos cerámicos.

Este tipo de materiales, hasta ahora fabricados de forma experimental en el Instituto de Cerámica de Galicia han dado lugar a la creación de una nueva empresa y a la actual construcción y puesta en funcionamiento, de una planta piloto valorada en 200.000.000 Ptas. ubicada en el norte de la Provincia de Lugo.

Dicha planta permitirá la fabricación de 20 Tm/día de material para su estudio y comercialización.
\end{abstract}

Palabras clave: Lodos Rojos, Barros Rojos, Acumuladores Térmicos, Ladrillos Densos, Proceso Bayer.

\section{Industrial obtaining of ceramic materials from the Bayer proces red mud's}

The Red Mud is the insoluble residue of the alumina extraction starting from the bauxite for the process Bayer. In Galicia they take place more than 400.000 annual tons of this industrial residue, constituted fundamentally by a mixture of oxides and iron hydroxides, titanium oxides, quartz, and small quantities of clays and other minerals.

The present work describes the process of industrial use of the Red Mud, to obtain dense bricks used in heat accumulators, arid for highways and other ceramic products.

This type of materials, up to now manufactured in an experimental way in the Institute of Ceramic of Galicia have given place to the creation of a new company and the current construction and setting in operation, of a pilot plant valued in 200.000 .000 Ptas. Located in the north of the Province of Lugo, this plant will allow the production of $20 \mathrm{Tm} /$ day of material for its study and commercialisation.

Keywords: Red Mud, Bauxite-Residues, Electric Thermal Storage, Dense Bricks, ETS

\section{INTRODUCCION}

En la producción de óxidos e hidróxidos de aluminio a partir de la bauxita por el proceso Bayer, las impurezas son separadas, generándose un residuo llamado Lodos o Barros Rojos. En este proceso se utiliza una solución caliente de hidróxido sódico para extraer la alúmina de la bauxita. La producción aproximada de residuos a nivel mundial es de 66,1 millones de toneladas anuales, el promedio anual de la planta de Alúmina Española S.A.-Alcoa Europe es de 400.000 Toneladas. Este residuo luego de ser lavado para recuperar alcalinos es vertido en embalses a la intemperie para su floculación. Los grandes volúmenes de residuos pueden crear problemas medioambientales debido a la ocupación de grandes áreas naturales y por el alto riesgo de contaminación del aire y del agua.

Se distinguen varias líneas de investigación dirigidas a este problema, agrupándose así:

Manejo de los vertidos: Métodos de clasificación, deshumidificación y lavado en la planta de producción. Sistemas de descarga y almacenamiento (floculación y secado) en las zonas de vertidos. Estudios del comportamiento de los Lodos Rojos en los embalses, como migración de elementos y minerales hacia el subsuelo y por lo tanto la posibilidad de contaminar corrientes acuáticas subterráneas. Estudios sobre la posible revegetación de los embalses, determinando especies vegetales resistentes a la alcalinidad del medio.

Reutilización de los vertidos: Utilización de los Lodos Rojos en la industria, como aditivo en las empresas de productos cerámicos y plásticos; producción de pigmentos y ladrillos sinterizados químicamente para la industria de la construcción; áridos para la construcción de carreteras ${ }^{[7]}$; baldosas acústicas. ${ }^{[5]}$ Utilización de los Lodos Rojos en agricultura, como agente acondicionador de suelos.

Reducción de los vertidos: Disminución del contenido de álcalis de los Lodos Rojos. Recuperación de elementos y minerales presentes en los Lodos, como, sodio, hierro, titanio, tierras raras, etc. ${ }^{[8]}$

Eliminación de los vertidos: Desarrollo de procesos libres de vertidos de Lodos Rojos.

Reciclado de los vertidos: Utilización de los Lodos Rojos en el mismo proceso Bayer.

Diversos autores encuentran más eficiente tanto a nivel ecológico como económico el reutilizar lodo rojo en produc- 
tos comerciales. ${ }^{[3,4,5]}$ Específicamente en España diversos departamentos de investigación han intentado responder a este reto científico. Se destacan por su utilidad en este trabajo las siguientes publicaciones: "Utilización cerámica de los barros rojos de las plantas de alúmina"[4]; "Obtención de ladrillos densos a partir de Barros Rojos, subproducto de las plantas de alúmina" ${ }^{\prime 9]}$, siendo posiblemente los pioneros en este tipo de aplicaciones. Ahora ha sido el momento preciso para retomar esas investigaciones y desarrollarlas hasta el escalado industrial, motivados por una conciencia ecológica y el interés del sector privado.

La facilidad con que la energía eléctrica se transforma en calor ha permitido desarrollar una gran variedad de sistemas y aparatos de calefacción, entre ellos están los calefactores por acumulación. Estos aparatos están diseñados para funcionar con un ciclo de carga y descarga separados en el tiempo, ésta característica permite aprovechar total o casi totalmente la tarifa eléctrica con discriminación de horas valle (tarifa nocturna). El almacenamiento de calor se realiza en un núcleo acumulador, compuesto por un conjunto de ladrillos de material refractario de elevado punto de fusión (del orden de $1600^{\circ} \mathrm{C}$ ) por lo que pueden alcanzar grandes temperaturas. El núcleo acumulador trabaja a una temperatura máxima entre 600 y $700^{\circ} \mathrm{C}$, temperatura que se ve limitada en razón de la temperatura que debe soportar el aislamiento y otras razones constructivas. Son materiales de alta densidad, aproximadamente $3 \mathrm{~g} / \mathrm{ml}$, con un calor específico del orden de $0,3 \mathrm{kcal} / \mathrm{kg}$. ${ }^{\circ} \mathrm{C}$, dependiendo de cada material. Estas características suponen un peso del núcleo acumulador de 5-6 Kg. por $\mathrm{kw} / \mathrm{h}$ de acumulación y capacidades de acumulación de 500 a $625 \mathrm{kwh} / \mathrm{m}^{3}$ para un salto térmico de 50 a $650^{\circ} \mathrm{C}$. Los materiales más utilizados son la periclasa en los acumuladores dinámicos y la magnetita en los estáti$\cos ^{[1]}$ Verificando lo expuesto en las publicaciones anteriores, este trabajo expone los resultados preliminares a la implementación de una planta piloto para la producción industrial de estos ladrillos.

\section{EXPERIMENTAL}

\subsection{Material}

La muestra de lodo rojo utilizada en este trabajo procede del depósito de la fabrica de Alúmina Española S.A.-Alcoa Europe, localizada en San Ciprián, provincia de Lugo, España.

\subsection{Caracterización}

\subsubsection{HUMEDAD DE RECEPCIÓN}

Este valor es variable pues es dependiente de las condiciones atmosféricas de la zona de vertidos, como de la profundidad a la cual es recogida la muestra. El material suministrado para las pruebas de laboratorio contó con contenido medio de humedad del $31 \% \pm 3$.

\subsubsection{PÉRDIDA POR CALCINACIÓN}

El lodo rojo al calcinarse a $1000^{\circ} \mathrm{C}$ por un tiempo de mantenimiento de una hora presenta una pérdida del $10 \% \pm 1$.

\subsubsection{DENSIDAD REAL}

Se determinó la densidad real con picnómetro de helio sobre muestra de lodo rojo seco, molido y tamizado por tamiz de $62 \mu \mathrm{m}$. Su valor es de $3,39 \mathrm{~g} / \mathrm{ml}$.

\subsubsection{ANÁLISIS QUÍMICO}

En las Tablas I y II se presentan los resultados de elementos mayoritarios y traza respectivamente.

TABLA I

ANÁLISIS POR FRX. ELEMENTOS MAYORITARIOS. PORCENTAJE EN ÓXIDO

\begin{tabular}{|lcccccccc|}
\hline Oxidos & $\mathbf{F e}_{2} \mathbf{O}_{\mathbf{3}}$ & $\mathbf{T i O}_{2}$ & $\mathrm{Al}_{2} \mathbf{O}_{\mathbf{3}}$ & $\mathbf{S i O}_{2}$ & $\mathbf{N a}_{2} \mathbf{O}$ & $\mathbf{C a O}$ & $\mathbf{M g O}$ & $\mathbf{K}_{2} \mathbf{O}$ \\
\hline Porcentaje & 30,6 & 23,3 & 20,5 & 7,0 & 4,75 & 4,65 & 0,17 & 0,05 \\
\hline
\end{tabular}

Tabla II

ANÁlisis POR FRX. ElEMENTOS TRAZA. PARTES POR MILLÓN

\begin{tabular}{|lccccccccccc|}
\hline Elemento & $\mathbf{Z r}$ & $\mathbf{C r}$ & $\mathbf{V}$ & $\mathbf{Y}$ & $\mathbf{S r}$ & $\mathbf{P b}$ & $\mathbf{Z n}$ & $\mathbf{C u}$ & $\mathbf{N i}$ & $\mathbf{C o}$ & $\mathbf{R b}$ \\
\hline ppm. & 3198 & 2737 & 1780 & 192 & 109 & 116 & 69 & 31 & 18 & 16 & $<5$ \\
\hline
\end{tabular}

\subsubsection{ANÁLISIS POR DRX}

Por difracción de Rayos- $X$ se detectan fases mineralógicas muy variadas y complejas presentando materiales amorfos en abundancia que se revelan por la altura del ruido de fondo y por la baja intensidad de las señales. Se definen, las siguientes fases mayoritarias en el lodo rojo seco a $110^{\circ} \mathrm{C}$ por 24 horas: Hematita, Gibbsita Boehmita, Rutilo Goetita y Cristobalita,

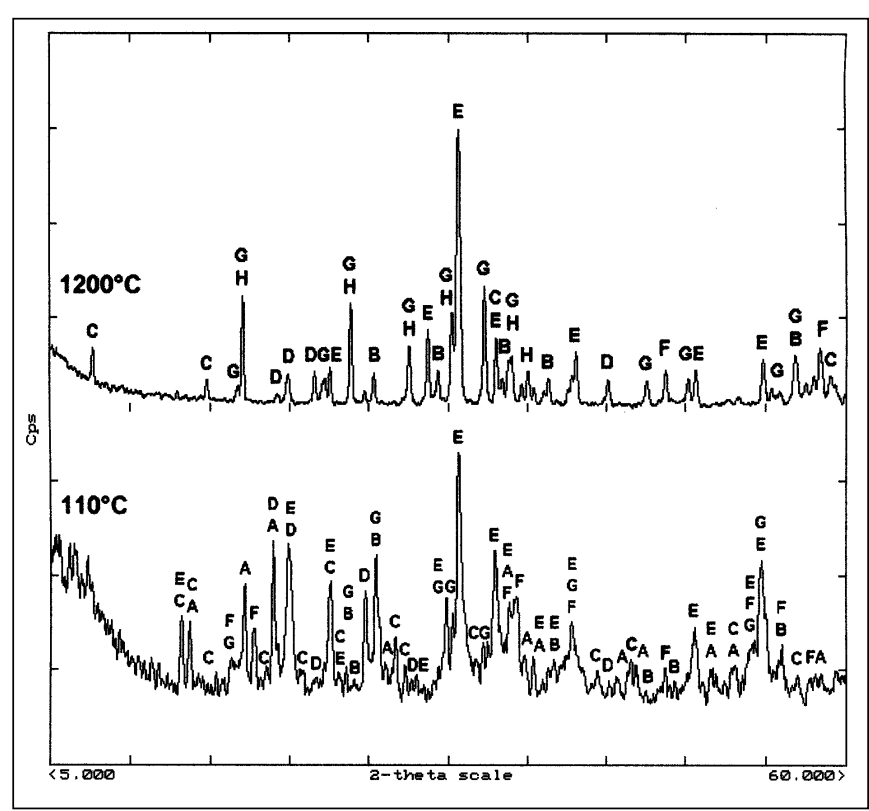

Fig. 1 Difractogramas de Lodo Rojo a $110^{\circ}$ C y $1200^{\circ}$ C. 
presentándose además en menor cantidad una serie de compuestos con elementos característicos como el Sodio y Calcio. A $1200^{\circ} \mathrm{C}$ las fases predominantes son Pseudobrookita Hematita y Titanatos de Hierro y Aluminio, con presencia de $\beta$ alúmina ( $\mathrm{Ca}, \mathrm{Na})$. En la Tabla III se presentan estas fases con la correspondiente identificación utilizada en la Figura 1.

\subsubsection{ANÁLISIS TÉRMICO}

Por ATD y ATG se presentan los siguientes efectos endotérmicos máximos con perdidas de peso correspondientes. En la Figura 2, se muestra la gráfica correspondiente al experimento y en la Tabla IV la identificación ${ }^{[6]}$ de las reacciones.

\subsubsection{MICROSCOPÍA DE CALEFACCIÓN}

Se realizó microscopía de calefacción con una rampa de calentamiento de $10^{\circ} \mathrm{C} /$ minuto hasta temperatura de fusión parcial. El resultado puede observarse en la Figura 3; en la Tabla V se enumeran los principales comportamientos.

\subsubsection{ENSAYOS DE COCCIÓN}

Se realizaron tratamientos térmicos para observar la evolución de los valores de densidad aparente y real sobre cilindros prensados isostáticamente. Las temperaturas de ensayo fueron $110,1000,1050,1100,1150,1200,1250$ y $1300^{\circ} \mathrm{C}$, con un tiempo de mantenimiento a temperatura máxima de 120 minutos y con rampa de subida de $5^{\circ} \mathrm{C} /$ minuto. Es importante señalar que a partir de $1200^{\circ} \mathrm{C}$ suele presentarse una ligera adherencia de la probeta al soporte, posiblemente originada por la presencia de una fase líquida. Los resultados se presentan en las Figuras 4 y 5, representando la influencia de la temperatura de calcinación frente al porcentaje de porosidad y frente a los valores de densidad aparente y real respectivamente.

Se concluye que a partir de $1000^{\circ} \mathrm{C}$ comienza una disminución de porosidad hasta $1150^{\circ} \mathrm{C}$ en donde es mínima. Se observa además que en el rango de $1150^{\circ} \mathrm{C}$ a $1200^{\circ} \mathrm{C}$ las temperaturas intermedias son más favorables que en el intervalo $1100^{\circ} \mathrm{C}$ a $1150^{\circ} \mathrm{C}$ en donde se presenta una pendiente más acusada. De los análisis realizados se concluye que los Lodos Rojos pueden procesarse a temperaturas entre $1100^{\circ} \mathrm{C}$ y $1200^{\circ} \mathrm{C}$ para obtener sinterizados que estarán constituidos por óxidos de hierro y titanio y titanatos de aluminio y hierro estables, con una densidad superior a $3 \mathrm{~g} / \mathrm{ml}$.

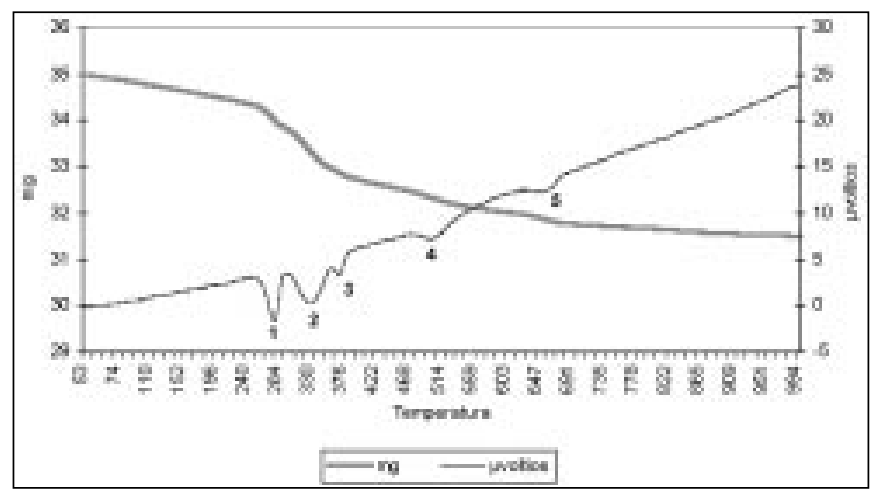

Fig. 2 Curvas de ATD y ATG del Lodo Rojo seco
TABLA III

FASES CRISTALINAS PRESENTES EN EL LODO ROJO

\begin{tabular}{|c|c|c|c|}
\hline Símbolo & $\begin{array}{c}\text { Elemento } \\
\text { característico }\end{array}$ & Fase cristalina & Fórmula \\
\hline \multirow[t]{2}{*}{$\mathbf{A}$} & \multirow[t]{2}{*}{ Aluminio } & Boehmita & $\mathrm{AlOOH}$ \\
\hline & & Gibbsita & $\mathrm{Al}(\mathrm{OH})_{3}$ \\
\hline B & Titanio & Rutilo y Brookita & $\mathrm{TiO}_{2}$ \\
\hline \multirow[t]{4}{*}{$\mathrm{C}$} & \multirow[t]{4}{*}{ Sodio } & Oxido de Titanio y Sodio & $\mathrm{Na}_{0.23} \mathrm{TiO}_{2}$ \\
\hline & & Natrosilita & $\mathrm{Na}_{2} \mathrm{Si}_{2} \mathrm{O}_{5}$ \\
\hline & & Diaoyudaoita & $\mathrm{NaAl}_{11} \mathrm{O}_{17}$ \\
\hline & & Sodalita & $\mathrm{Na}_{4} \mathrm{Al}_{3} \mathrm{Si}_{3} \mathrm{O}_{12} \mathrm{Cl}$ \\
\hline D & Sílice & Cristobalita, Cuarzo y Tridimita & $\mathrm{SiO}_{2}$ \\
\hline \multirow[t]{4}{*}{$\mathbf{E}$} & \multirow[t]{4}{*}{ Hierro } & Hematita y Magemita & $\mathrm{Fe}_{2} \mathrm{O}_{3}$ \\
\hline & & Siderita & $\mathrm{FeCO}_{3}$ \\
\hline & & Oxido de Hierro & $\mathrm{Fe}_{3} \mathrm{O}_{4}$ \\
\hline & & Lepidocrocita y Goetita & $\mathrm{FeO}(\mathrm{OH})$ \\
\hline \multirow[t]{5}{*}{$\mathbf{F}$} & \multirow[t]{5}{*}{ Calcio } & Oxido cálcico & $\mathrm{CaO}$ \\
\hline & & Mayenita & $\mathrm{Ca}_{12} \mathrm{Al}_{14} \mathrm{O}_{33}$ \\
\hline & & Aluminato cálcico & $\mathrm{CaAl}_{2} \mathrm{O}_{4}$ \\
\hline & & \begin{tabular}{|l|} 
Silicato dicálcico \\
\end{tabular} & $\mathrm{Ca}_{2} \mathrm{SiO}_{4}$ \\
\hline & & Oxido de Hierro y Calcio & $\mathrm{CaFe}_{2} \mathrm{O}_{4}$ \\
\hline \multirow[t]{4}{*}{ G } & \multirow[t]{4}{*}{ Hierro y Titanio } & Ilmenita & $\mathrm{FeTiO}_{3}$ \\
\hline & & Ilmenorutilo & $F e_{x}(\mathrm{Nb}, \mathrm{Ta})_{2 x} \mathrm{Ti}_{1-x} \mathrm{O}_{2}$ \\
\hline & & Ulvospinela & $\mathrm{Fe}_{2} \mathrm{TiO}_{4}$ \\
\hline & & \begin{tabular}{|l|} 
Pseudobrookita \\
\end{tabular} & $\mathrm{FeTiO}_{5}$ \\
\hline $\mathbf{H}$ & $\begin{array}{c}\text { Hierro + Titanio + } \\
\text { Aluminio }\end{array}$ & Titanatos de Aluminio y Hierro & $\mathrm{TiO}_{2}\left(\mathrm{Al}_{2} \mathrm{O}_{3}\right)_{1-x}\left(\mathrm{Fe}_{2} \mathrm{O}_{3}\right)_{x}$ \\
\hline
\end{tabular}

TABLA IV

REACCIONES OBSERVADAS EN ATD / ATG

\begin{tabular}{|c|c|l|}
\hline $\begin{array}{c}\mathbf{N}^{\circ} \text { de pico } \\
1\end{array}$ & $\begin{array}{c}\text { Temp. }\left({ }^{\circ} \mathbf{C}\right) \\
273\end{array}$ & $\begin{array}{l}\text { Reacción } \\
\text { Deshidratación de la Goetita } \alpha-\mathrm{FeO}(\mathrm{OH})\end{array}$ \\
\hline 2 & 325 & $\begin{array}{l}\text { Pérdida parcial o total de los grupos }(\mathrm{OH}) \\
\text { de la Gibbsita } \mathrm{Al}(\mathrm{OH})_{3}\end{array}$ \\
\hline 3 & 366 & $\begin{array}{l}\text { Deshidratación de la Limonita } \\
\alpha-\mathrm{FeO}(\mathrm{OH}) \cdot n \mathrm{H}_{2} \mathrm{O}\end{array}$ \\
\hline 4 & 498 & Deshidratación de la Boehmita $\gamma-\mathrm{AlO}(\mathrm{OH})$ \\
\hline 5 & 657 & Descarbonatación de la Siderita $\mathrm{FeCO}_{3}$ \\
\hline
\end{tabular}

TABLA V

COMPORTAMIENTO del Lodo Rojo EN Microscopía de CALEFACCióN

\begin{tabular}{|lc|}
\hline Comportamiento & Temperatura \\
\hline Estable & $20-1020^{\circ} \mathrm{C}$ \\
\hline Comienzo de la contracción & $1020^{\circ} \mathrm{C}$ \\
\hline Fin de la contracción - inicio de la dilatación & $1250^{\circ} \mathrm{C}$ \\
\hline Fin de la dilatación - inicio del reblandecimiento & $1300^{\circ} \mathrm{C}$ \\
\hline Fusión & $1373^{\circ} \mathrm{C}$ \\
\hline
\end{tabular}

TABLA VI

PROPIEDADES Y CONDICIONES DE LA MEJOR MEZCLA DE LODOS ROJOS CALCINADOS Y ARCILLA

\begin{tabular}{|l|c|}
\hline Mezcla & $\begin{array}{c}\mathbf{7 5 \%} \text { Lodo Rojo a } \mathbf{1 0 0 0}^{\circ} \mathbf{C} \\
\mathbf{+} \\
\mathbf{2 5 \%} \text { de arcilla }\end{array}$ \\
\hline Agua de amasado & $22 \%$ \\
\hline Temperatura de cocción & $1150^{\circ} \mathrm{C}$ \\
\hline Contracción & $13,4 \% \pm 0,2$ \\
\hline Absorción de agua & 2,71 \\
\hline Resistencia & $43 \mathrm{MPa}$ \\
\hline Densidad aparente & 2,76 \\
\hline
\end{tabular}

Boletín de la Sociedad Española de Cerámica y Vidrio. Vol. 38 Núm. 3 Mayo-Junio 1999 


\subsubsection{ENSAYOS DE CONFORMACIÓN}

Examinando los diferentes sistemas de conformación convencionales de materiales cerámicos y después de evaluar sus propiedades se consideró que para las condiciones dimensionales de la pieza final, los sistemas más apropiados son, el moldeo plástico y el prensado de polvos.

\subsubsection{Moldeo plástico}

Se determinó el límite líquido y el límite plástico. Los resultados son 30,7\% para el límite líquido y 25,8 para el límite plástico, siendo entonces su índice de plasticidad de $4,9 \%$. Aunque este índice se consideró muy ajustado, se realizaron pruebas en una extrusionadora de avance manual con una boquilla de sección cuadrada de $1 \mathrm{~cm}$ de lado.

Se observó que el lodo rojo extrusionado dentro de sus límites plásticos, no posee la resistencia adecuada para una producción industrial. Se aprecia también una diferencia del porcentaje de humedad entre las piezas extrusionadas al inicio de la carga con las del final del 7\%, quedando en algunos casos agua residual no integrada a la masa, y presentándose en las piezas extruidas al final grietas producidas por esta perdida. Las piezas que presentaron un mejor comportamiento fueron sometidas a calcinación a $1175^{\circ} \mathrm{C}$ en horno eléctrico, determinándose una contracción del $20 \% \pm 1$.

Los ensayos realizados con diferentes mezclas de material crudo con porcentajes variables de material calcinado y gra-
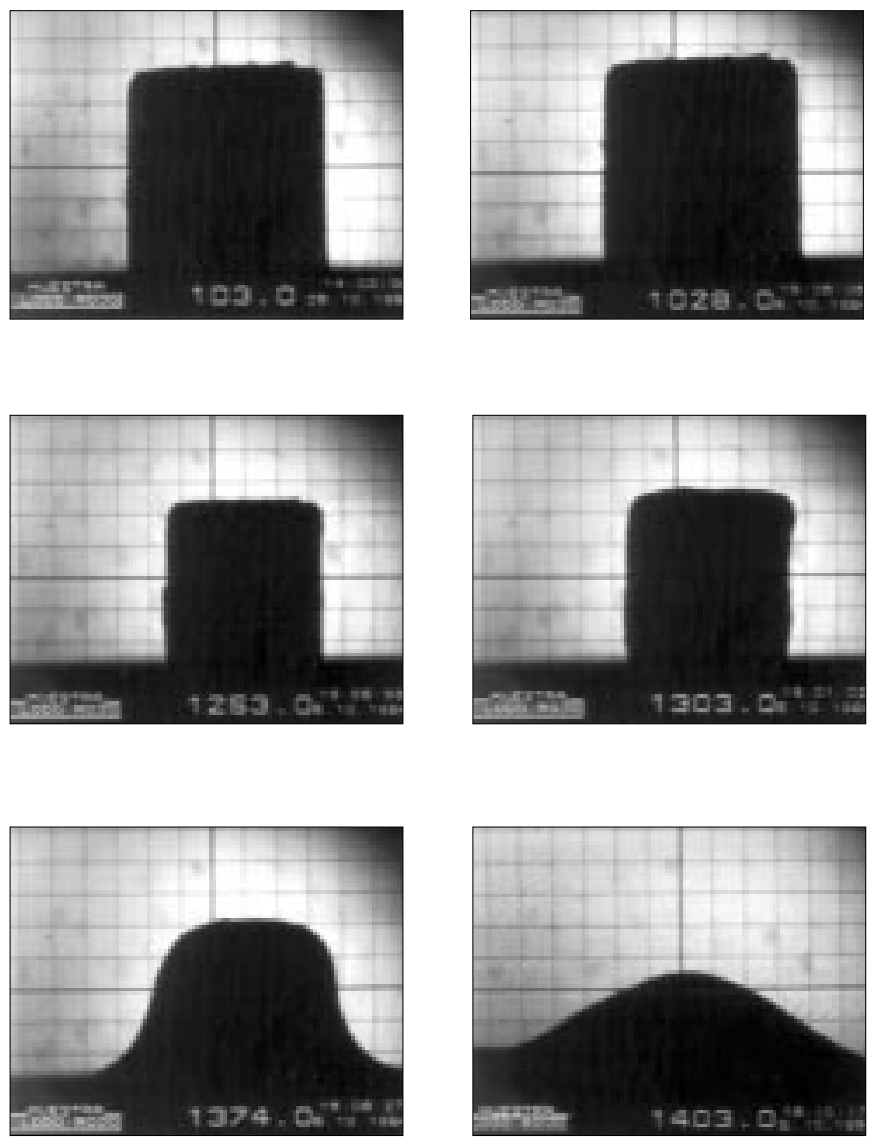

Fig. 3. Microscopía de calefacción de los Lodos Rojos. nulometrías medias $(<500 \mu \mathrm{m})$ se obtuvieron también resultados negativos. Se conformaron también mezclas de material calcinado y arcilla plástica, en un intento de mejorar su comportamiento en la extrusión y reducir su contracción. Se observa un mejor comportamiento en extrusión, sin ser excesivamente el deseable. El resultado más relevante es el de la mezcla presentada en la Tabla VI, en donde se exponen las características alcanzadas.

\subsubsection{Prensado de polvos}

Se realizaron diferentes pruebas con el material crudo únicamente y material crudo mezclado con varios porcentajes de material calcinado con granulometría media $(<500 \mu \mathrm{m})$, utilizando moldes para piezas cilíndricas de $5 \mathrm{~cm}$ de diámetro. Posteriormente, considerando los resultados anteriores y los requerimientos dimensionales del producto final (bloques de 20x20×5), se determinó que el proceso de conformación adecuado es el prensado de polvos. Con el fin de obtener una mejor aproximación a las condiciones del producto final, se utilizó un molde de acero con un volumen máximo de capacidad de $2340 \mathrm{~cm}^{3}$, con una superficie de carga de $10 \times 19,5 \mathrm{~cm}$. Como resultado de esta fase se observa que para obtener una mejor resistencia del producto en verde y una compactación superior es necesaria la adición de agua como ligante entre un 7 y un $10 \%$. Después de ensayar diferentes distribuciones granulométricas para empaquetamientos máximos ideales, el desarrollo matemático con mejores resultados fue el desarrollado por Andreasen. El resultado medio de esta serie experi-

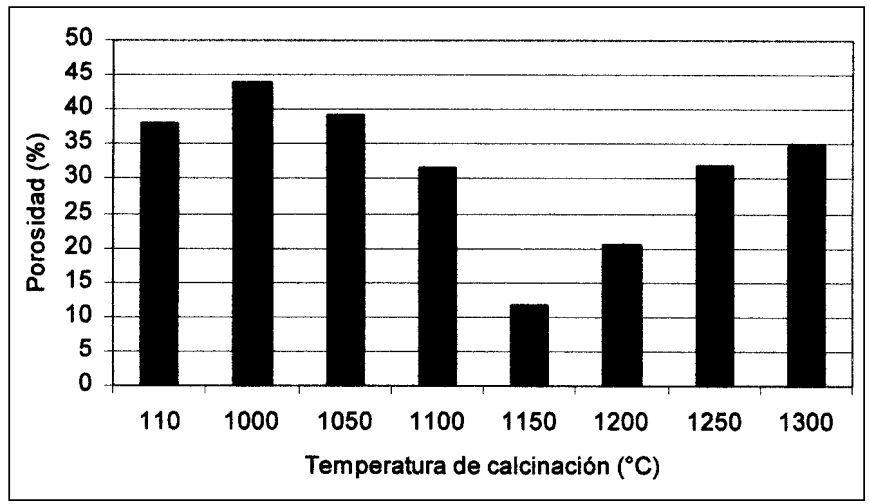

Fig. 4. Influencia de la temperatura de la porosidad

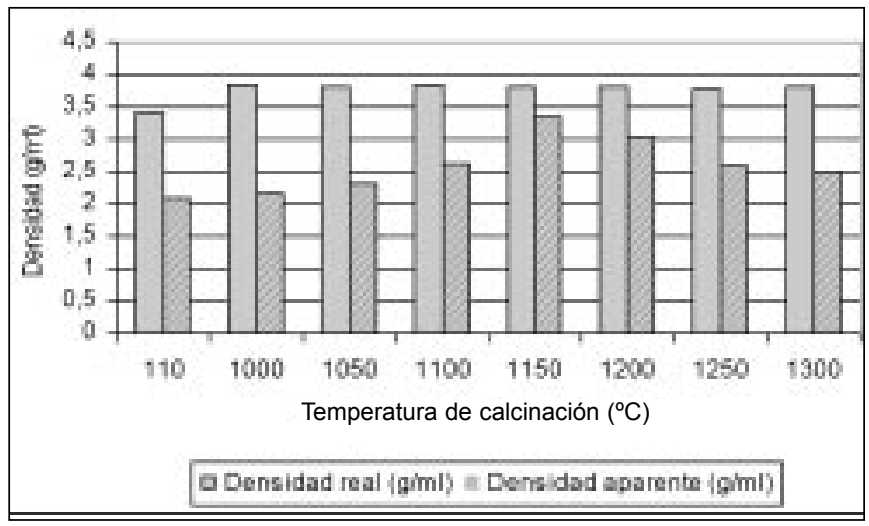

Fig. 5. Influencia de la temperatura en la densidad. 
mental se presenta en la Tabla VII.

Posteriormente se realizaron ensayos para determinar la influencia de diferentes variables en el producto final (velocidad de prensado, tiempo de mantenimiento, métodos de humectación y mezclado de las diferentes fracciones, tiempo y temperatura de secado, tiempos y velocidades de calcinación y enfriamiento, etc.) llegándose de esta forma al proceso de conformación definitivo para piezas de $10 \times 19,5 \times 5 \mathrm{~cm}$ en el laboratorio. Una descripción de este proceso se presenta a continuación.

\subsection{Proceso}

Para la elaboración de los ladrillos en laboratorio, la conformación de las piezas se dividió en las siguientes etapas:

\subsubsection{PREPARACIÓN DEL POLVO}

Se determina que la granulometría de partida del material crudo seco estará comprendida dentro de los siguientes tamaños:

Fracción 1: $<3>1 \mathrm{~mm}$. Fracción 2: $<1 \mathrm{~mm}$. Fracción 3: $<250 \mu \mathrm{m}$.

Se partió del material con la humedad de recepción (31\%) y se extruyó utilizando una boquilla circular de $3 \mathrm{~mm}$ de diámetro. Este proceso reduce drásticamente los tiempos de secado y facilita las operaciones posteriores. Una vez seco el material se granula y se tamiza. Debido a la fragilidad del material extruido este proceso se realizó directamente sobre el tamiz de $3 \mathrm{~mm}$, consiguiéndose simultáneamente fracciones inferiores. Se calcinaron separadamente las fracciones 1 y 2 en horno eléctrico a una temperatura máxima de $1150^{\circ} \mathrm{C}$ y tiempo de mantenimiento de 60 minutos, las cuales una vez finalizado el ciclo, se vuelven a tamizar para obtener las fracciones ya mencionadas. Se realizó este tamizado doble porque algunas partículas alcanzan a adherirse a las otras, y su separación es más sencilla al tener fracciones de tamaño más concretas. La fracción 3 se deja como material crudo.

Con este chamotado se alcanza la estabilidad química y mecánica del Lodo Rojo y a su vez se disminuyen los posibles problemas de secado y contracción de cocido de los ladrillos.

\subsubsection{ADICIÓN DEL LIGANTE}

Se decide que en esta etapa de laboratorio, no se utilice ningún aglomerante comercial con el fin de determinar la fiabilidad de este proceso en condiciones desfavorables, por lo tanto

TABLA VII

PROPIEDADES Y CONDICIONES DE LA MEZCLA PRELIMINAR

\begin{tabular}{|l|c|}
\hline Mezcla & $\begin{array}{c}\text { Lodo calcinado a } 1150^{\circ} \mathrm{C}: \\
14 \%(<3>2 \mathrm{~mm}), 19 \%(<2>1 \mathrm{~mm}), \\
15 \%(<1>0,5 \mathrm{~mm}), 26 \%(<500 \mu \mathrm{m}) \\
\text { Lodo crudo: } 26 \%(<500)\end{array}$ \\
\hline Contracción & 4,5 en largo y ancho: 9,5 en espesor \\
\hline Absorción & $4,3 \%$ \\
\hline Densidad & $2,97 \mathrm{~g} / \mathrm{ml}$ \\
\hline Presión & $550 \mathrm{~kg} / \mathrm{cm}^{2}$ \\
\hline Temperatura de cocción & $1175^{\circ} \mathrm{C} / 240$ minutos \\
\hline
\end{tabular}

para este fin solo se utilizará agua. El mezclado del material con el ligante (7\% en peso del total del material), fue realizado usando un tambor giratorio similar al sistema de molino de bolas.

Los porcentajes de cada fracción en peso son:

Fracción 1: 30\%. Fracción 2: 45\%. Fracción 3: 25\%

El procedimiento de mezcla utilizado fue el siguiente: Mezclado 1: la fracción 1 con el total del ligante, Mezclado 2 adición de la fracción 2 y finalmente Mezclado 3 adición de la fracción 3. Todas las operaciones de mezcla se realizan hasta lograr homogeneización.

Ejemplo para obtener bloques de 10x19x5cm.

Peso total del material calcinado y crudo seco: 3000g., repartido de la siguiente forma: fracción 1: 900g, fracción 2: 1350g y fracción 3: $750 \mathrm{~g}$. Peso de agua utilizada como ligante $210 \mathrm{~g}$. Tiempos de mezclado: Todas las mezclas se realizaron con un tiempo promedio de 10 minutos.

\subsubsection{LLENADO DEL MOLDE}

Se utilizó el molde descrito con anterioridad. El material se vertió en la cavidad del molde y con ayuda de una espátula se repartió sin presionar hasta obtener una superficie superior llana. Posteriormente se carga el pistón macho, realizando pequeños desplazamientos multidireccionales para ajustar el pistón y el material.

\subsubsection{PRENSADO}

Se utilizó una prensa hidráulica con carga máxima de 120 Ton. El prensado se realizó de la forma más lenta posible ejecutándolo en "tres tiempos", es decir a 210, 420 y $630 \mathrm{Kg} / \mathrm{cm}^{2}$, se detuvo la carga y se realizó una descarga de $100 \mathrm{Kg} / \mathrm{cm}^{2}$ para permitir el desplazamiento de las partículas, continuando hasta el siguiente valor. Cuando se alcanzó la presión máxima del equipo, se mantuvo la carga por 60 segundos y posteriormente se descargó totalmente.

\subsubsection{DESCARGA DE LA PIEZA}

Debido a la configuración del equipo, las piezas se desmoldaron colocando el molde sobre unos soportes adecuados y ejerciendo nuevamente presión sobre el pistón en la misma dirección de carga. En la parte inferior fue colocada una superficie amortiguadora para eliminar o disminuir la posibilidad de fractura de la pieza al caer.

\subsubsection{MANEJO DEL PRODUCTO EN VERDE}

El producto se colocó sobre un soporte de cordierita de dimensiones apropiadas para su transporte. En este momento los ladrillos húmedos son sometidos a dimensionamiento $\mathrm{y}$ pesados para los cálculos posteriores.

\subsubsection{SECADO}

El secado de los ladrillos se realizó en estufa eléctrica semiindustrial, a una temperatura de $100^{\circ} \mathrm{C}$, con un tiempo de permanencia de 24 horas. 


\subsubsection{COCCIÓN:}

La cocción se realizo en horno eléctrico a una temperatura máxima de $1175^{\circ} \mathrm{C}$, con un tiempo de mantenimiento de 240 minutos.

\section{RESULTADOS Y COMPARACIÓN FRENTE A UN LADRILLO COMERCIAL}

En la Tabla VIII se presentan los resultados de diferentes determinaciones sobre un ladrillo comercial y el obtenido en laboratorio: Densidad real, aparente y porcentaje de porosidad, (por porosimetría de mercurio). Resistencia al choque térmico, (norma B.S.1902/PART 1A, 1966; British Standard Specification). Dilatometría en el rango $20-800^{\circ} \mathrm{C}$. Calor específico. Composición mayoritaria. Poder de acumulación. Precio aproximado de los ladrillos. Relación precio frente a poder acumulativo.

En la Figura 6 se presenta el resultado de la prueba de cesión de calor de los dos ladrillos en ambiente abierto sobre un intervalo de temperatura de $500-50^{\circ} \mathrm{C}$.

En el laboratorio se prepararon series de ladrillos que fueron enviados a Technotherm GmbH (empresa alemana líder en el mercado de calefactores por acumulación) para comprobación y evaluación de las piezas obtenidas por este procedimiento, consiguiendo la homologación para ser utilizados en los acumuladores ensamblados por su empresa filial en Galicia (Electromecánicos Viveiro S.A.). Se realizo una pre-serie de 100 ladrillos con la colaboración de la empresa Lomba Camiña S.A., confirmándose la efectividad del método propuesto.

\section{CONCLUSIONES}

Cuando se selecciona un material o materiales para la fabricación de un producto comercial generalmente se tienen en cuenta tres puntos importantes:

a) Que el material tenga o desarrolle las propiedades físicas y mecánicas deseadas.

TABLA VIII

COMPARACIÓN DE PROPIEDADES LADRILLO COMERCIAL Y LADRILLO ICG

\begin{tabular}{|c|c|c|}
\hline Propiedades & $\begin{array}{l}\text { Ladrillo Comercial } \\
\text { Ref.Art. 830-900-006 }\end{array}$ & Ladrillo ICG \\
\hline Densidad real $(\mathrm{g} / \mathrm{ml})$ & 4,39 & 3,74 \\
\hline Densidad aparente $(\mathrm{g} / \mathrm{ml})$ & 3,76 & 3,28 \\
\hline Porosidad (\%) & 16,55 & 12,18 \\
\hline Resistencia a la compresión & $105,4 \mathrm{MPa}$ & $171,8 \mathrm{MPa}$ \\
\hline Resistencia a la flexión & $80,7 \mathrm{Kg}$ & $283,55 \mathrm{Kg}$ \\
\hline Resistencia al choque térmico & 19 ciclos & +21 ciclos \\
\hline Dilatación $20-800^{\circ} \mathrm{C}\left(\times 10^{-6}{ }^{\circ} \mathrm{C}^{-1}\right)$ & 9,8 & 8,0 \\
\hline $\mathrm{Cp} \mathrm{cal} / \mathrm{g}{ }^{\circ} \mathrm{C}$ & 0,20 & 0,19 \\
\hline Composición Mayoritaria & $\begin{array}{l}\text { Oxidos de hierro } \\
\text { Fase vítrea }\end{array}$ & $\begin{array}{c}\text { Oxidos de hierro } \\
\text { Titanatos de hierro } \\
\text { Fase vítrea }\end{array}$ \\
\hline Poder de acumulación (\%) & 100 & 87,3 \\
\hline Ptas./Ladrillo 20x20x5 cm & 425 & 80 \\
\hline $\mathrm{R}=$ Precio $/ \mathrm{P} . \mathrm{A}$ & 4,25 & 0,91 \\
\hline
\end{tabular}

b) Que el material pueda ser procesado o manufacturado en la forma requerida.

c) Que tanto el material como el proceso sean económicos.

En la medida que estos puntos son alcanzados, se obtendrá un producto competitivo y comercial. [2]

Basados en las diferentes fases experimentales brevemente expuestas en este trabajo, se puede concluir que este proyecto cumple satisfactoriamente con estos tres puntos. Recapitulando tenemos que: El Lodo Rojo principalmente por su composición mineralógica desarrolla después de su calcinación bajo condiciones apropiadas, unas propiedades térmicas que le permiten ser un serio competidor frente a los productos comerciales que en la actualidad en su gran mayoría vienen de mercados extranjeros. Después de su conformación bajo las condiciones anteriormente descritas el producto final presenta valores superiores y mejores comportamientos frente su resistencia al choque térmico, resistencia a flexión y compresión, por ejemplo. Siendo en otros aspectos muy similar al producto comercial. El Lodo Rojo permite ser conformado con facilidad por prensado de polvos, bajo las condiciones anteriormente expuestas, sin embargo, como la gran mayoría de los procesos, éste también será susceptible de mejorar, un ejemplo puede ser la necesidad de adicionar un ligante-lubricante comercial de uso común en las empresas del sector cerámico tradicional para permitir trabajar piezas de mayor tamaño a las realizadas en el laboratorio, siendo éste un condicionante de gran importancia para el éxito comercial. Otras series de ajustes serán necesarios desde el momento de la puesta en marcha de la planta industrial para obtener la máxima eficiencia del proceso y una alta reproducibilidad en las características del producto final, hasta ahora conseguidas en el trabajo de laboratorio. El material desde un primer punto de vista puede considerarse bastante económico debido principalmente a que es un residuo del proceso Bayer. Al desarrollarse este proceso desde su inicio con la condicionante de utilizar tecnología convencional de procesos cerámicos tradicionales, es fácil asegurar por lo tanto que la inversión necesaria para el montaje de una planta industrial para el sinterizado del Lodo Rojo y su posterior conformación, como en este caso concreto, en ladrillos acumuladores de calor, no necesitara de inversiones excesivas. Garantizando además que el personal necesario para su fabricación no requiera algún nivel de conocimiento demasiado avanzado.

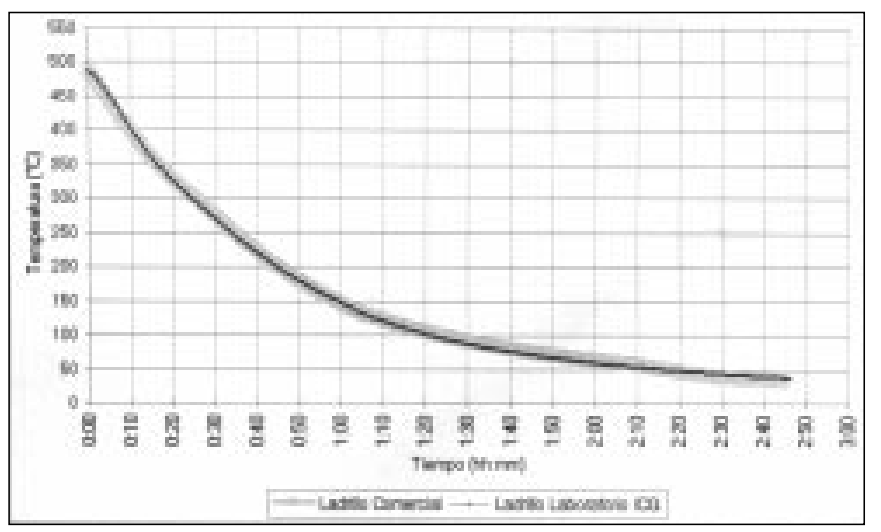

Fig. 6. Influencia de la temperatura en la densidad 


\section{PLANTA PILOTO}

El desarrollo de este tipo de materiales a partir de los Lodos Rojos hasta ahora fabricados de forma experimental en el Instituto de Cerámica de Galicia, ha motivado a diferentes sectores de la sociedad a involucrarse más seriamente con el proyecto, impulsando su realización a nivel industrial. Partiendo de este procedimiento que está protegido por dos patentes de invención registradas a nombre de la Universidad de Santiago, y adquiridas por la empresa Indebar S.A. (Investigación y Desarrollo de Barros Rojos S.L.). Esta empresa está construyendo en la actualidad una planta piloto en las inmediaciones de Cervo (Lugo) con una inversión cercana a 300 millones de Ptas. Dicha planta permitirá la fabricación entre 15 y 20 Tm/día de material para su comercialización y estudio de nuevas aplicaciones, siendo cofinanciado por la Xunta de Galicia, el CDTI e Indebar S.A..

\section{BIBLIOGRAFIA}

1. Alonso Andonegui, A.; Fernández Salazar, L. C.; de Landa Amezua, J.; Toledano Gasca, J. C.; del Valle Antépara, J. “Calefacción Eléctrica, Confort, Cálculo y Sistemas". Ed. Paraninfo. Madrid. (1996).

2. Askelan, D. R. "The science and engineering of materials - $2^{\text {nd }}$ SI ed.". Ed. Chapman and Hall. UK. (1990)

3. McCarthy, S., Armour-Brown, A., Iyer, V. S., Desu, S. B., Kander, R. G., and Vaseashta, A. "Utilization of Jamaica bauxite tailings as a building material and its socio-economic considerations" presentado en "Proceedings of An international bauxite tailings workshop" 2-6 de noviembre de 1992. Australia occidental.

4. Morales, F.; Moya, J. S.; De Aza, S. “Obtención de ladrillos densos a partir de Barros Rojos, subproducto de las plantas de alúmina" Actas II del VIII CongresoExposición Argentino y II iberoamericano de Cerámica, Vidrio y Refractarios. 14-18 de noviembre de 1988. Buenos Aires.

5. Pepplinkhouse, H. J., Davern, W. A. "Acoustic tiles from solid wastes" Journal of the Australian Ceramic Society. Vol.11. №2. (1995).

6. Todor D. N. "Thermal analysis of minerals" Abacus Press. Tunbridge Wells, Kent. (1976)

7. The Australian bauxite and alumina producers. "Proceedings of An international bauxite tailings workshop" 2-6 de noviembre de 1992. Australia occidental.

8. Wagh, A. S.; Morrison, D. E. Utilization of Jamaican red mud - an overview" presentado en "Proceedings of An international bauxite tailings workshop" 2-6 de noviembre de 1992. Australia occidental.

Recibido: $21-10-98$

Aceptado: 1-3-99 